Case Report

\title{
Rapid Ascending Sensorimotor Paralysis, Hearing Loss, and Fatal Arrhythmia in a Multimorbid Patient due to an Accidental Overdose of Fluoxetine
}

\author{
Matthew Herrmann, Prissilla Xu, and Antonio Liu \\ White Memorial Medical Center Department of Internal Medicine, Los Angeles, CA, USA \\ Correspondence should be addressed to Matthew Herrmann; herrmann.matthew7@gmail.com
}

Received 27 April 2017; Revised 29 August 2017; Accepted 10 September 2017; Published 16 October 2017

Academic Editor: Peter Berlit

Copyright (c) 2017 Matthew Herrmann et al. This is an open access article distributed under the Creative Commons Attribution License, which permits unrestricted use, distribution, and reproduction in any medium, provided the original work is properly cited.

\begin{abstract}
Background. Common side effects of selective serotonin reuptake inhibitors (SSRIs) include tachycardia, drowsiness, tremor, nausea, and vomiting. Although SSRIs have less toxic side effects compared to more traditional antidepressants, serious and life threatening cases of SSRI overdose have been reported. We describe a 24 -year-old multimorbid female who presented to the emergency department with rapid onset ascending sensorimotor paralysis, complicated by respiratory and cardiac arrest, found to have fatal levels of fluoxetine by toxicological analysis, not taken in a suicidal act. Results. Autopsy was performed at the Los Angeles County Medical Examiner's Office of a female with no evidence of traumatic injury. Toxicological analysis revealed lethal levels of fluoxetine, toxic levels of diphenhydramine, and multiple other coingested substances at nontoxic levels. Neuropathological examination of the brain and spinal cord revealed no evidence of Guillain-Barre paralysis. Conclusions. Lethal levels of fluoxetine and multiple potential drug-to-drug interactions in our patient likely contributed to her unique signs and symptoms. This is the first case reporting neurologic signs and symptoms consisting of rapid onset ascending sensorimotor paralysis, hearing loss, respiratory failure, cardiac arrest, and death in a patient with lethal levels of fluoxetine.
\end{abstract}

\section{Introduction}

Selective serotonin reuptake inhibitors (SSRIs) are commonly used to treat depression and anxiety disorders and are some of the most widely prescribed antidepressants today [1]. Multiple case studies have reported that SSRIs are associated with a less toxic side effect profile and fewer reported deaths have been attributed to overdose compared to more traditional antidepressants, such as tricyclic antidepressants $[2,3]$; however, serious and life threatening sequelae have been reported. Additionally, among SSRIs, fluoxetine has been reported to be the least toxic by hazard index measures [3]. The largest published case series on fluoxetine overdoses found that the most common effects were signs of serotonin syndrome such as tachycardia, drowsiness, tremor, nausea, and vomiting [4]. Other significant sequelae include seizures, cardiac toxicity, and death $[2,5]$. We report abnormal symptomatology of a young woman due to an accidental fatal fluoxetine overdose, consisting of rapid onset, ascending sensorimotor paralysis, bilateral hearing loss, respiratory failure, cardiac arrest, and eventual death.

\section{Case Report}

A 24-year-old Hispanic female with diabetes mellitus type 1, anemia, hypertension, chronic pancreatitis with partial pancreatectomy, cholecystectomy, and splenectomy presented to the emergency department (ED) with abdominal pain not relieved by oral pain medications. She recalled her home medications to be mirtazapine $30 \mathrm{mg}$ orally nightly, fluoxetine unknown dose orally three times a day, carbamazepine $200 \mathrm{mg}$ orally twice daily, insulin glargine $15-20$ units subcutaneously nightly, morphine sulfate $30 \mathrm{mg}$ orally twice daily, tramadol $50 \mathrm{mg}$ orally four times a day, hydromorphone $4 \mathrm{mg}$ orally six times a day as needed for pain, and hydrocodone/acetaminophen $325 / 10 \mathrm{mg}$ orally six times a day as needed for pain, and she had been taking them to relieve her pain. She was discharged from the ED but 
returned on the same day with worsening abdominal pain, loss of sensation in lower extremities, lips, and hands, and ascending paralysis. The patient was noted to have a leukocytosis with bandemia, fever, and tachycardia suspicious for sepsis. Computed tomography (CT) of the abdomen was significant for small bowel edema and ascites. The patient continued to deteriorate with worsening ascending paralysis, bilateral hearing loss, hypotension, and respiratory failure with subsequent endotracheal intubation. She underwent two rounds of cardiopulmonary resuscitation (CPR) for a total of approximately 109 minutes but ultimately expired.

An autopsy was performed and documented a welldeveloped woman with no evidence of traumatic injuries. Postmortem serum analysis revealed fatal levels of heart blood fluoxetine concentration of $2.3 \mathrm{mcg} / \mathrm{mL}$.

The cause of death was thought to be multiple medication intoxication with fatal levels of fluoxetine. A neuropathologist was consulted who agreed with the diagnosis and thought that her symptoms were primarily due to overmedication with fluoxetine. She had no known history of suicidal ideation or attempt but did have chronic pain. Thus, the favored mode of death was accident. Contributory factors to death included acute on chronic, culture negative pancreatitis with abscess formation, and likely sepsis.

\section{Experimental}

3.1. Specimens. All specimens were analyzed and collected at autopsy at Los Angeles County Medical Examiner's Office. Submitted specimens included heart ventricles and septum, left lung, right lung, liver, right kidney, small bowel and colon, and head of pancreas. The neuropathological specimens consisted of formalin fixed brain, spinal cord with attached dura mater, and complete cranial dura mater.

\section{Results}

Positive findings on gross and microscopic pathology revealed evidence of chronic pancreatitis with superimposed acute pancreatitis with abscess, culture negative for three days after death, and surgical absent pancreatic tail. The spleen and gall bladder were also surgically absent. The cut surface of the pancreas was pale, fibrotic, and gritty, with loss of normal lobular appearance. Rare small punctate hemorrhages were present, with a single $\sim 0.5 \mathrm{~cm}$ possible abscess cavity present centrally in fibrotic area with no communication to the surface. Other microscopic findings revealed acute pneumonia of the left lung, mild emphysematous changes of bilateral lungs, few hypertrophic myocytes of the heart septum and left ventricle, and mild nonspecific chronic hepatitis. There was no evidence of septic emboli.

Gross impression of neuropathological specimens revealed brain swelling, cavum septum pellucidum, external rotation of left hippocampus, beaking of the inferior frontal lobe, cerebral vermis atrophy, and no gross atrophy of the nerves and spinal cord, with no spinal cord lesions noted on cross section. The meninges were clear and there was no gross evidence of meningitis. Neuropathological examination of the brain and spinal cord revealed no evidence of Guillain-Barre paralysis and findings consistent with hypoxic-ischemic encephalopathy. The findings of cerebellar atrophy and hippocampal neuronal dropout suggested a chronic seizure disorder, which was not noted on history.

4.1. Toxicological Analysis. Toxicology tests revealed a lethal level of fluoxetine, $1.8 \mathrm{mcg} / \mathrm{mL}$, in the femoral blood, and $2.3 \mathrm{mcg} / \mathrm{mL}$ in the heart blood, as well as $0.81 \mathrm{mcg} / \mathrm{mL}$ of norfluoxetine in the femoral blood and $1.1 \mathrm{mcg} / \mathrm{mL}$ of norfluoxetine in the heart blood. Diphenhydramine was also found at toxic levels, $0.78 \mathrm{mcg} / \mathrm{mL}$, in the femoral blood. Other medications present at nontoxic levels included metoclopramide, mirtazapine, nortramadol, tramadol, midazolam, lidocaine, carbamazepine, hydrocodone, codeine, morphine, and hydromorphone in her heart and femoral blood. Levels of ethanol, barbiturates, cocaine and metabolites, fentanyl, methamphetamine and methlenedioxymethamphetamine, free phencyclidine, free oxycodone, and free oxymorphine were not detected

\section{Discussion}

Fluoxetine, one of the SSRI antidepressants, was introduced into clinical practice over 25 years ago and has remained one of the most popular and safe [6-8] antidepressants in the United States [9]. While approximately half of fluoxetine intoxications remain asymptomatic [4, 9], symptoms of fluoxetine overdose are "minimally toxic" and include tachycardia, drowsiness, tremor, nausea, and vomiting [10]. However, more serious sequelae have also been reported, such as seizures [10-16], cardiac conduction abnormalities [16], CNS depression, respiratory arrest, and even death [5]. Our patient whose toxicological analysis revealed lethal levels of fluoxetine presented with tachycardia and, uniquely, loss of sensation in her lower extremities, lips, and hands and developed rapidly ascending paralysis with respiratory arrest, cardiac arrest, and eventual death.

The patient reported taking fluoxetine, unknown dose, three times a day despite the recommended dose of between 20 and $80 \mathrm{mg}$ per day [17]. It was unclear for how long she had been taking this dose. There was no indication from her history or from the pathologist's report that this was a suicidal attempt. Mode of death determined to be accidental from multiple medication intoxication. The toxicologist report revealed multiple drugs present in her femoral and heart blood, with diphenhydramine, fluoxetine, and norfluoxetine at toxic levels. Our review of the literature revealed that our patient presented with neurologic findings that are first to be described in association with fluoxetine ingestion.

The pathomechanism by which toxic levels of fluoxetine could have caused these unique symptoms is described in, in vitro, animal and human studies. Multiple experimental studies have showed that at hypertherapeutic and overdose concentrations of fluoxetine, fluoxetine demonstrates evidence of cytotoxicity, antiproliferative effects, and mitochondrial dysfunction. Specifically, these studies noted inhibition of mitochondrial function and depletion of cellular ATP levels with significantly increased lactate production, activation of 


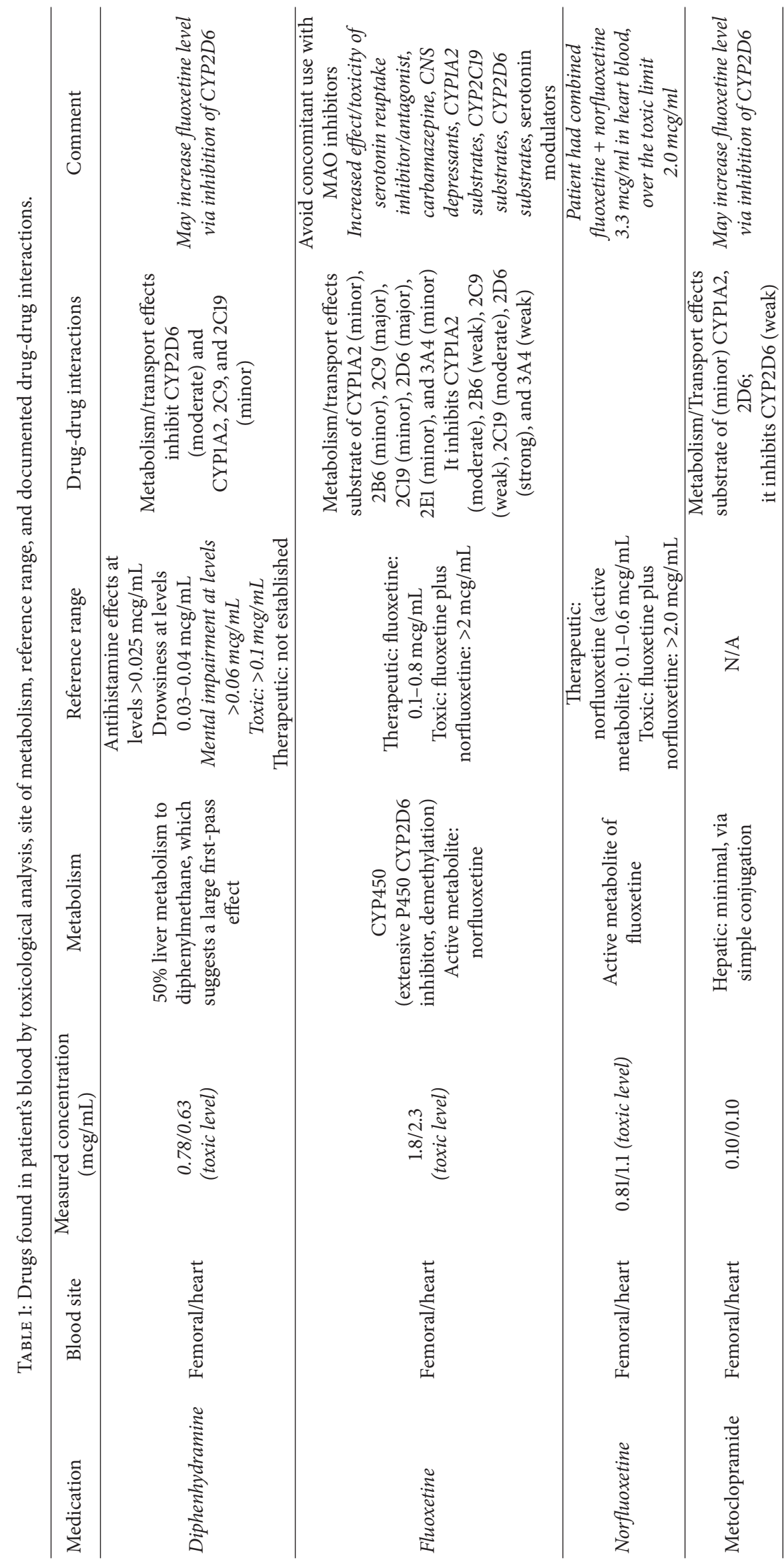




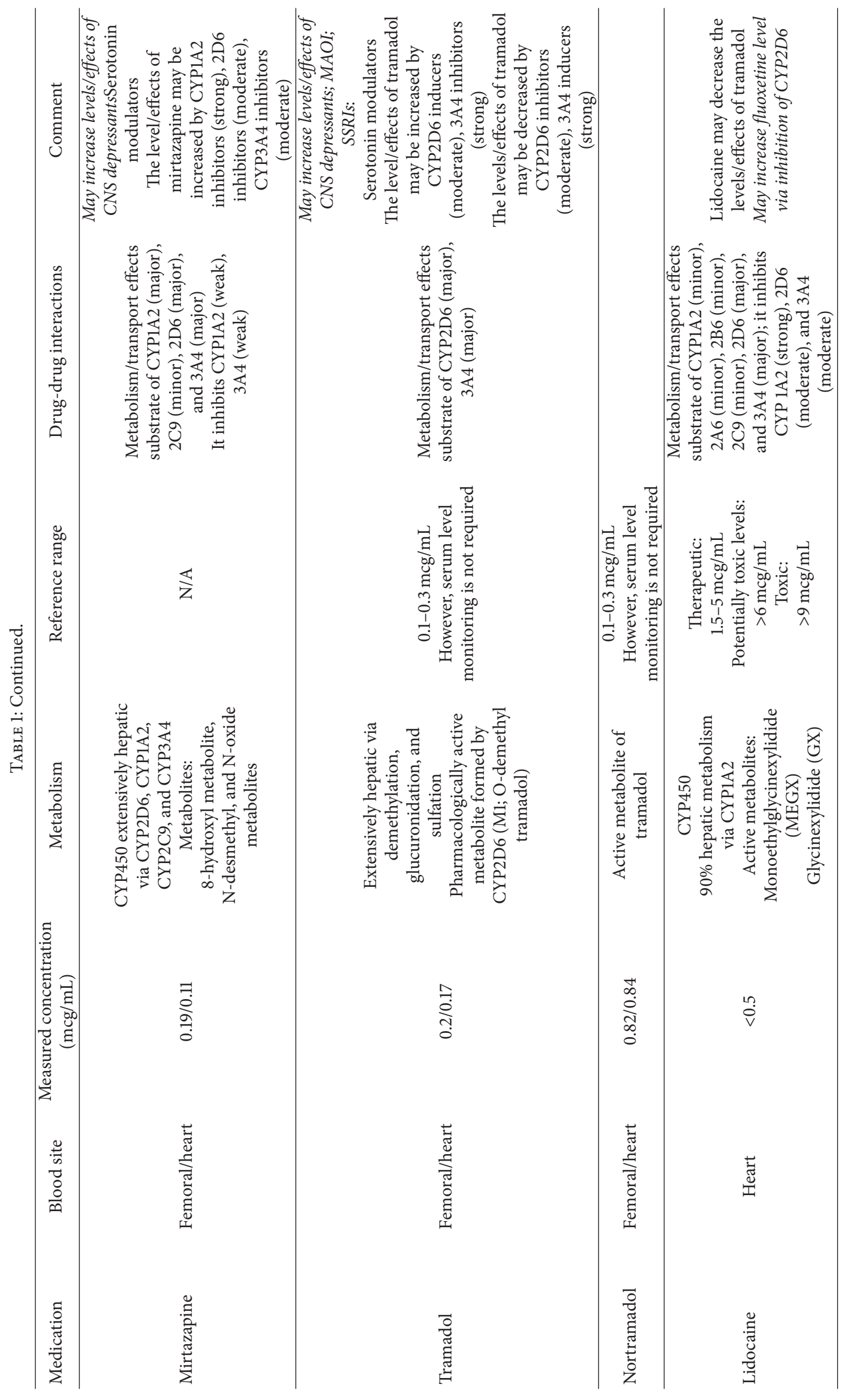




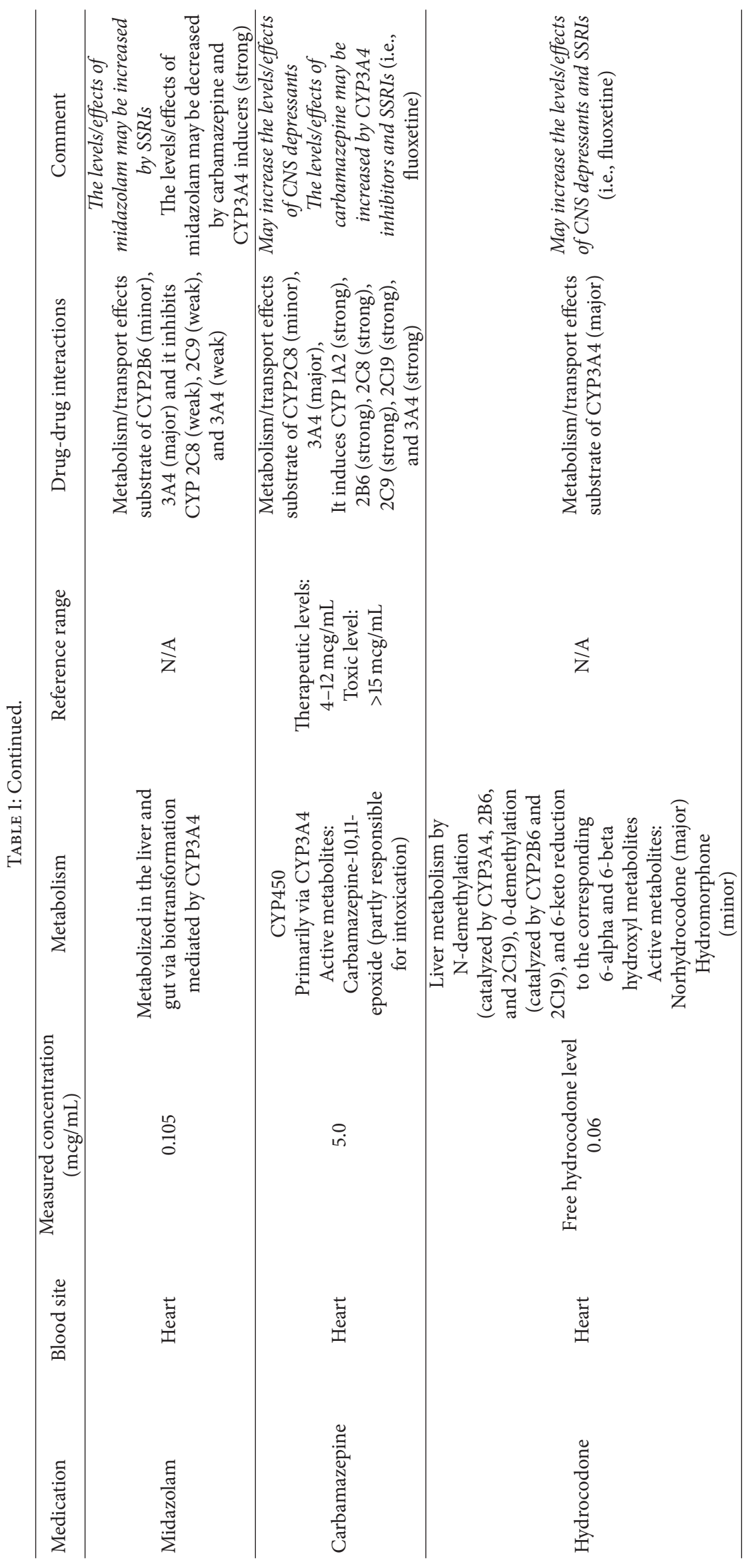




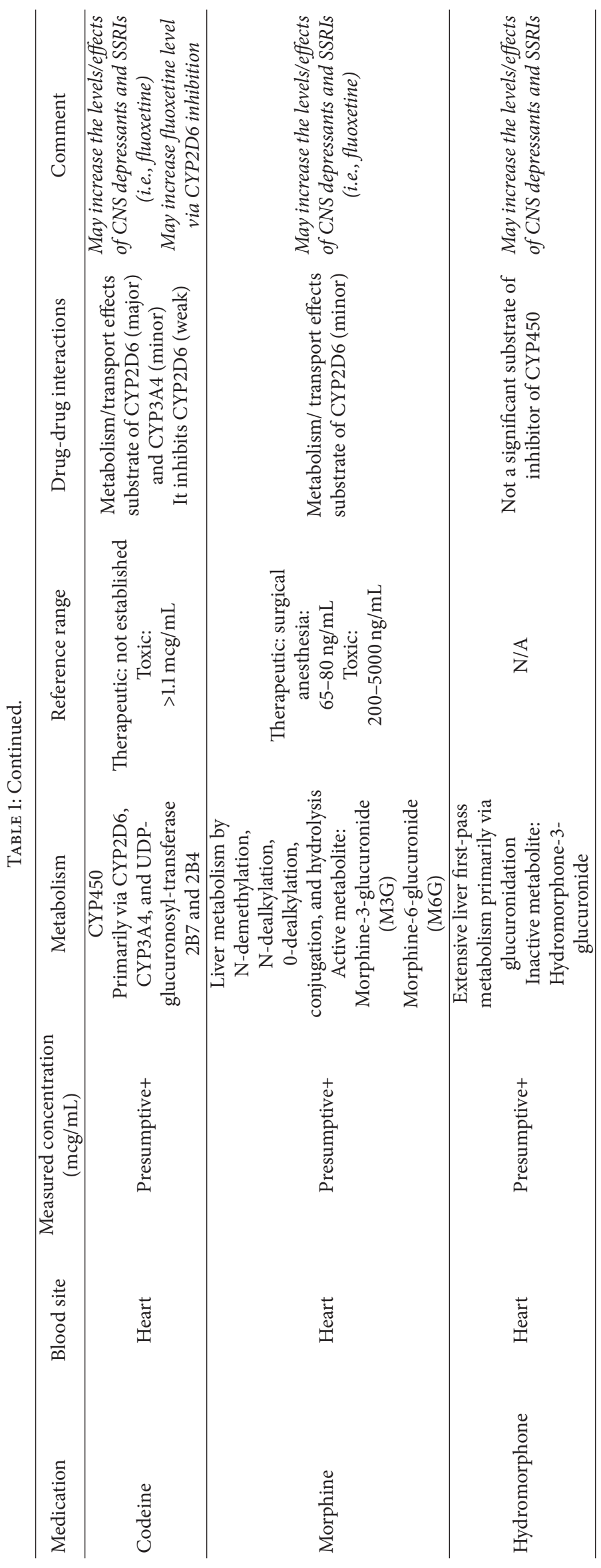


apoptosis, and evidence of redox stress and DNA damage [1822].

The drug-drug interactions which potentially contributed to our patient's toxic levels of fluoxetine and unique symptomatology include fluoxetine with metoclopramide, carbamazepine, tramadol, mirtazapine, codeine, hydrocodone, and morphine. Fluoxetine, diphenhydramine, and metoclopramide are all inhibitors CYP2D6, one of the cytochrome P450 enzymes necessary for detoxification of foreign chemicals and metabolism of drugs. Inhibitor of this enzyme likely contributed to the toxic levels noted in her blood. Also, it is well documented that the concurrent use of fluoxetine with codeine, hydrocodone, hydromorphone, mirtazapine, morphine, and tramadol, and the use of concurrent carbamazepine with codeine, hydromorphone, and mirtazapine may result in increased risk of serotonin syndrome [2325]. Overall, our patient's unique symptomatology could be explained by the damaging effects of toxic levels of fluoxetine as well as the multiple drug-drug interactions as outline in Table 1.

Most fatalities due to fluoxetine exposure are reported either with extremely large doses (greater than 150 times the daily dose) or with the presence of coingestants such as ethanol or benzodiazepines $[4,6]$. In 2014, the NDPS reported that the range of fluoxetine blood concentrations in listed fatalities ranged from $0.68 \mathrm{mcg} / \mathrm{ml}$ to $2.2 \mathrm{mcg} / \mathrm{L}$, which is significantly greater than the typical steady state therapeutic concentration of $0.25 \mathrm{mcg} / \mathrm{mL}$. To put this into perspective, toxicological analysis on our patient revealed fatal levels of heart blood fluoxetine and norfluoxetine concentrations at $2.3 \mathrm{mcg} / \mathrm{mL}$ and $1.1 \mathrm{mcg} / \mathrm{mL}$, respectively, well above the therapeutic limit. It is important to note that, in one study of antidepressant overdose, no correlation between fluoxetine level and mental status was found, while another study of fluvoxamine overdose found no correspondence between drug levels and symptom severity $[17,18]$, suggesting that each patient presenting with an SSRI overdose, despite quantity consumed, can have unique symptomatology.

Although most patients recover from fluoxetine overdose, high dose ingestions can lead to cardiovascular and neurologic complications, including death. In this case, the patient had lethal levels of fluoxetine noted in her blood, as well as levels of multiple other medications with known drugdrug interactions to fluoxetine, possibly contributing to her unique symptomatology. However, this patient's presenting neurologic signs and symptoms are unique and have yet to be described in current reports of fatal levels of fluoxetine ingestions or coingestions involving fluoxetine.

\section{Conflicts of Interest}

The authors have no conflicts of interest to report.

\section{References}

[1] S. H. Preskorn, H. P. Feighner, C. Y. Stanga, and R. Ross, Antidepressants: Past, Present and Future, 2004, Past, Present and Future.
[2] G. K. Isbister, S. J. Bowe, A. Dawson, and I. M. Whyte, "Relative toxicity of selective serotonin reuptake inhibitors (SSRIs) in overdose," Journal of Toxicology-Clinical Toxicology, vol. 42, no. 3, pp. 277-285, 2004.

[3] N. White, T. Litovitz, and C. Clancy, "Suicidal antidepressant overdoses: a comparative analysis by antidepressant type.," Journal of medical toxicology : official journal of the American College of Medical Toxicology, vol. 4, no. 4, pp. 238-250, 2008.

[4] D. J. Borys, S. C. Setzer, L. J. Ling, J. J. Reisdorf, L. C. Day, and E. P. Krenzelok, "Acute fluoxetine overdose: A report of 234 cases," American Journal of Emergency Medicine, vol. 10, no. 2, pp. 115120, 1992.

[5] M. W. Lai, W. Klein-Schwartz, G. C. Rodgers et al., "2005 Annual report of the American association of poison control centers' national poisoning and exposure database," Clinical Toxicology, vol. 44, no. 6-7, pp. 803-932, 2006.

[6] J. T. Barbey and S. P. Roose, "SSRI safety in overdose," Journal of Clinical Psychiatry, vol. 59, suppl. 15, pp. 42-48, 1998.

[7] H. A. Spiller, S. Morse, and C. Muir, "Fluoxetine ingestion: a one year retrospective study," Veterinary and Human Toxicology, vol. 32, article 153, 1990.

[8] W. Chiang, M. Ford, P. Wax, R. Hoffman, M. Howland, and L. Goldfrank, "Prospective evaluation of fluoxetine ingestions," Veterinary and Human Toxicology, vol. 32, article 348, 1991.

[9] Top 200 generic drugs by unit in 2007. Drug Topics, June 2017, http://drugtopics.modernmedicine.com/drug-topics/news/ modernmedicine/modern-medicine-feature-articles/top-200generic-drugs-unit-2007.

[10] J. J. Weber, "Seizure activity associated with fluoxetine therapy," The Journal of Clinical Pharmacology, pp. 8:296-8:296, 1989.

[11] R. Hargrave, D. Martinez, and A. J. Bernstein, "Fluoxetineinduced seizures," Psychosomatics, vol. 33, no. 2, pp. 236-237, 1992.

[12] V. P. Prasher, "Seizures associated with fluoxetine therapy," Seizure, vol. 2, no. 4, pp. 315-317, 1993.

[13] M. R. Ware and R. B. Stewart, "Seizures Associated with Fluoxetine Therapy," DICP, vol. 23, no. 5, pp. 428-428, 2016.

[14] G. Braitberg and S. C. Curry, "Seizure after isolated fluoxetine overdose," Annals of Emergency Medicine, vol. 26, no. 2, pp. 234237, 1995.

[15] R. Gross, P. N. Dannon, E. Lepkifker, J. Zohar, and M. Kotler, "Generalized seizures caused by fluoxetine overdose," American Journal of Emergency Medicine, vol. 16, no. 3, pp. 328-329, 1998.

[16] A. Graudins, C. Vossler, and R. Wang, "Fluoxetine-induced cardiotoxicity with response to bicarbonate therapy," American Journal of Emergency Medicine, vol. 15, no. 5, pp. 501-503, 1997.

[17] G. Gartlehner, R. A. Hansen, L. C. Morgan et al., "Comparative benefits and harms of second-generation antidepressants for treating major depressive disorder: An updated meta-analysis," Annals of Internal Medicine, vol. 155, no. 11, pp. 772-785, 2011.

[18] E. Elmorsy, A. Al-Ghafari, F. M. Almutairi, A. M. Aggour, and W. G. Carter, "Antidepressants are cytotoxic to rat primary blood brain barrier endothelial cells at high therapeutic concentrations," Toxicology in Vitro, vol. 44, pp. 154-163, 2017.

[19] A. L. Hansson, Z. Xia, M. C. Berglund, A. Bergstrand, J. W. Depierre, and L. Nässberger, "Reduced cell survival and morphological alterations induced by three tricyclic antidepressants in human peripheral monocytes and lymphocytes and in cell lines derived from these cell types," Toxicology in Vitro, vol. 11, no. 1-2, pp. 21-31, 1997. 
[20] T. S. Davies and W. M. Kluwe, "Erratum: Preclinical toxicological evaluation of sertraline hydrochloride (Drug and Chemical Toxicology)," Drug and Chemical Toxicology, vol. 21, no. 4, pp. 521-537, 1998.

[21] A. Carvajal Garcia-Pando, J. Garcia del Pozo, A. Sanchez et al., "Hepatoxicity associated with new antidepressants," Journal of Clinical Psychiatry, pp. 135-137, 2002.

[22] Y. Li, L. Couch, M. Higuchi, J.-L. Fang, and L. Guo, "Mitochondrial dysfunction induced by sertraline, an antidepressant agent," Toxicological Sciences, vol. 127, no. 2, pp. 582-591, 2012.

[23] C. Lacy, L. Armstrong, M. P. Goldman, and L. L. Lance, Drug Information Handbook, 20th edition.

[24] http://www.micromedexsolutions.com.

[25] http://www.globalrph.com. 


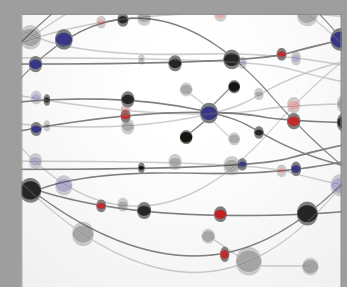

The Scientific World Journal
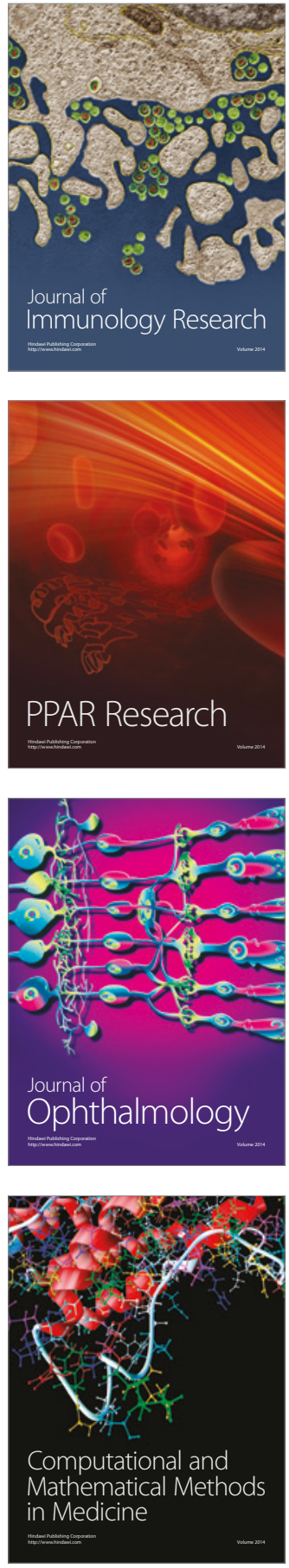

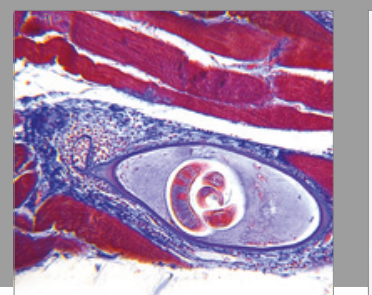

Gastroenterology Research and Practice
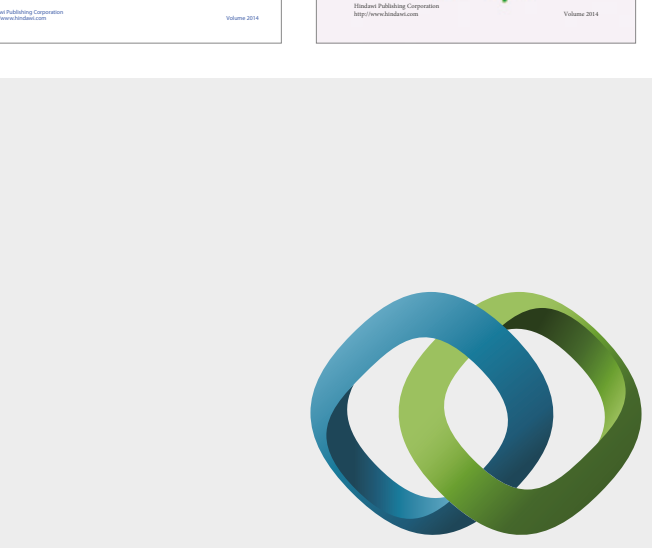

\section{Hindawi}

Submit your manuscripts at

https://www.hindawi.com
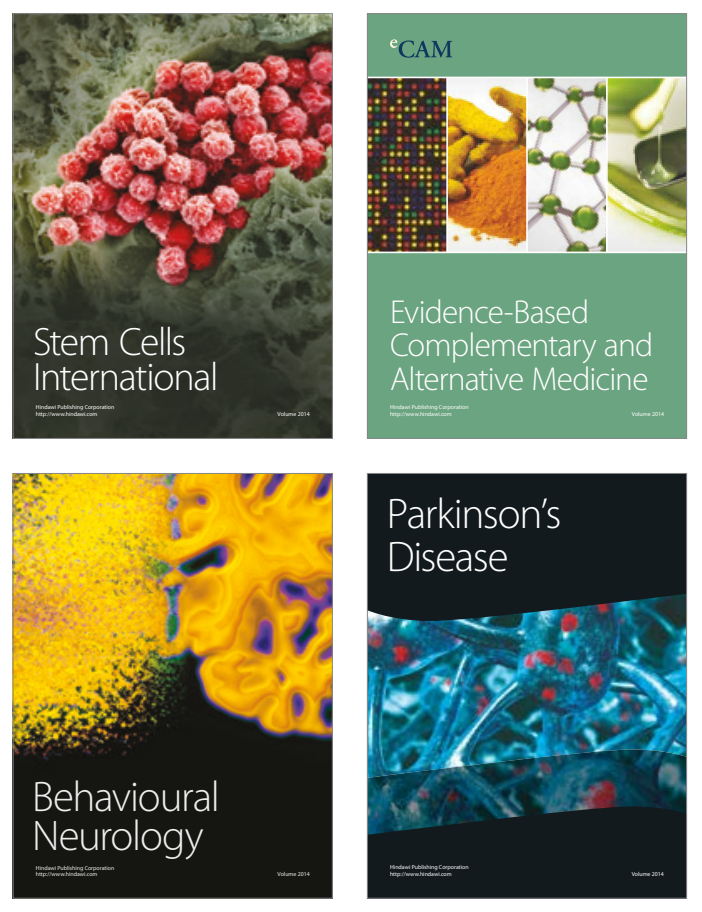
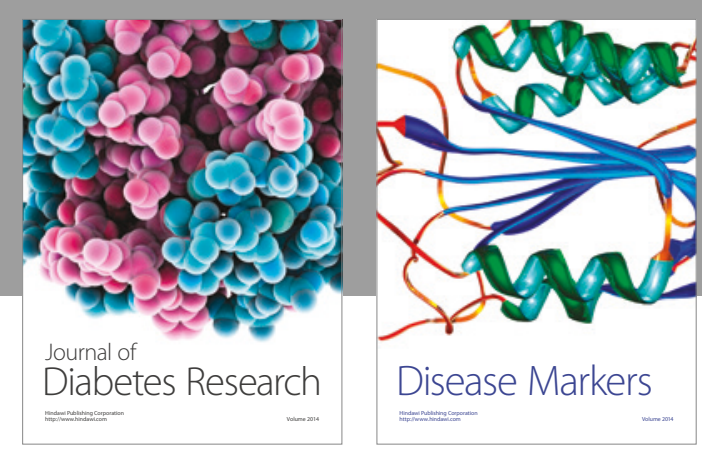

Disease Markers
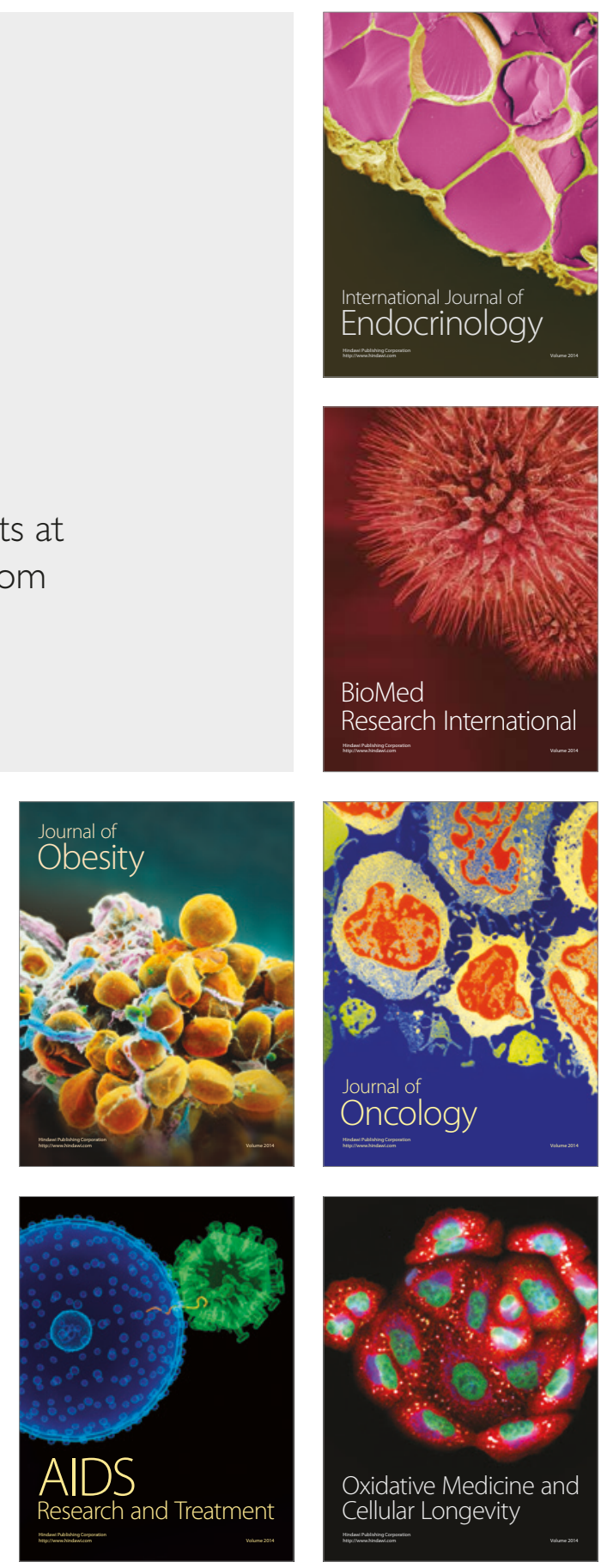